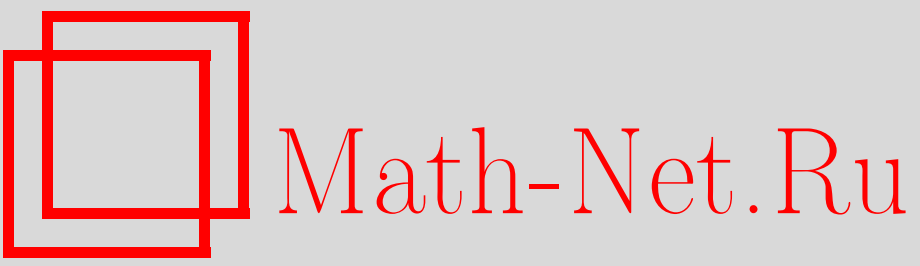

В. И. Войтицкий, Н. Д. Копачевский, О нормальных колебаниях маятника с полостью, частично заполненной идеальной несжимаемой жидкостью, Итоги науки и техн. Сер. Соврем. мат. и ее прил. Темат. обз., 2021, том 190, 34-49

DOI: https://doi.org/10.36535/0233-6723-2021-190-34-49

Использование Общероссийского математического портала Math-Net.Ru подразумевает, что вы прочитали и согласны с пользовательским соглашением

http://www.mathnet.ru/rus/agreement

Параметры загрузки:

IP: 3.82 .47 .9

26 апреля 2023 г., 05:07:04 


\title{
О НОРМАЛЬНЫХ КОЛЕБАНИЯХ МАЯТНИКА \\ С ПОЛОСТЬЮ, ЧАСТИЧНО ЗАПОЛНЕННОЙ ИДЕАЛЬНОЙ НЕСЖИМАЕМОЙ ЖИДКОСТЬЮ
}

\author{
(c) 2021 г. $\quad$ В. И. ВОЙТИЦКИЙ, Н. Д. КОПАЧЕВСКИЙ
}

\begin{abstract}
АннотАция. Рассматривается линейная начально-краевая задача, порожденная проблемой малых движений пространственного маятника с полостью, частично заполненной однородной несжимаемой жидкостью, в случае, когда момент силы трения в сферическом шарнире пропорционален угловой скорости. Приводится операторная трактовка задачи, устанавливается теорема о сильной разрешимости задачи Коши на конечном отрезке времени. Для соответствующей спектральной задачи доказывается дискретность спектра и его локализация в полосе, устанавливается степенная асимптотика собственных значений, суммируемость системы корневых элементов методом Абеля-Лидского.
\end{abstract}

Ключевые слова: начально-краевая задача, гильбертово пространство, самосопряженный линейный оператор, дискретный спектр, базисность по Абелю-Лидскому.

\section{NORMAL OSCILLATIONS OF A PENDULUM WITH A CAVITY PARTIALLY FILLED WITH AN IDEAL INCOMPRESSIBLE FLUID}

\author{
(c) 2021
}

\author{
V. I. VOYTITSKY, N. D. KOPACHEVSKY
}

\begin{abstract}
We consider a linear initial-boundary-value problem generated by the problem of small motions of a spatial pendulum with a cavity partially filled with a homogeneous incompressible fluid, in the case where the moment of friction forces in the spherical hinge is proportional to the angular velocity. We propose an operator interpretation of the problem and prove a theorem on the strong solvability of the Cauchy problem on a finite time interval. For the corresponding spectral problem, the discreteness of the spectrum and its localization in a strip are proved, power asymptotics of eigenvalues ??are found, and the summability of the system of eigenvectors is established by the Abel-Lidsky method.
\end{abstract}

Keywords and phrases: initial-boundary-value problem, Hilbert space, self-adjoint linear operator, discrete spectrum, Abel-Lidsky basis property.

AMS Subject Classification: 70E55, 35M33

1. Введение. Проблемы движения твердых тел с жидким наполнением изучаются с середины прошлого столетия многими известными математиками. В простейшем случае, когда полость

Работа Н. Д. Копачевского выполнена при поддержке Министерства образования и науки РФ (проект № 14.Z50.31.0037). 
внутри твердого тела полностью заполнена идеальной несжимаемой жидкостью, движение системы (гиростата) эквивалентно движению твердого тела с измененными характеристиками. Данный результат принадлежит Н. Е. Жуковскому (см. [11]) и основан он на введении так называемых потенциалов Жуковского - стационарных функций, являющихся решениями вспомогательных краевых задач, зависящих лишь от формы полости.

Если полость внутри тела заполнена частично, то гидромеханическая система имеет подвижный центр масс, обусловленный наличием свободной движущейся поверхности, что существенно усложняет задачу описания движения. Такие проблемы изучались в линейной постановке, начиная с работ Н. Н. Моисеева (см. [25]), отметим также статьи Л. Н. Сретенского, Г. С. Нариманова, Д. Е. Охоцимского (см. [26, 29, 30,33]), а также более поздние монографии [24, 27, 28, 32, 34, 35].

В работе С. Г. Крейна и Н. Н. Моисеева [20] для исследования проблемы малых движений твердого тела, содержащего жидкость со свободной поверхностью, впервые применялись методы функционального анализа и теории линейных операторов, действующих в гильбертовом пространстве. Результаты этой статьи отражены в более поздних монографиях [17,36, 37]. Данная работа посвящена более подробному изучению соответствующих спектральных проблем и написана в продолжение исследований С. Г. Крейна и Н. Д. Копачевского. Отметим, что исследование проведено на основе статьи Н. Д. Копачевского [14], в которой использована та же операторная схема (основанная на эквивалентных преобразованиях кинематических краевых условий на свободной поверхности). В этой работе можно найти доказательства ряда утверждений, которые здесь приводятся без доказательства. В данном исследовании более точно описаны спектральные свойства задачи в отсутствие трения и, кроме того, изучено влияние трения в шарнире на локализацию и асимптотику собственных значений, а также на базисность системы корневых элементов.

Отметим, что используемый в задаче операторный подход использовался в предыдущих работах авторов $[7,8,16]$, где изучались проблемы малых движений сочлененных маятников с жидким наполнением. При этом оказалось, что он одинаково применим как для идеальных, так и для вязких жидкостей, частично либо целиком заполняющих полости, а также для систем несмешивающихся жидкостей в полостях (случай сочлененных гиростатов изучался в серии более ранних работ Н. Д. Копачевского и Э. И. Батыра, см., например, [4,5]). Весь класс описанных задач в операторной форме сводится к изучению абстрактной задачи Коши в сумме гильбертовых пространств $H=H_{1} \oplus H_{2}$ :

$$
\begin{gathered}
\left(\begin{array}{cc}
C_{1} & 0 \\
0 & g C_{2}
\end{array}\right) \frac{d}{d t}\left(\begin{array}{l}
z_{1} \\
z_{2}
\end{array}\right)+ \\
\left(\begin{array}{cc}
A_{1} & 0 \\
0 & 0
\end{array}\right)\left(\begin{array}{l}
z_{1} \\
z_{2}
\end{array}\right)+g\left(\begin{array}{cc}
0 & B_{12} \\
B_{21} & 0
\end{array}\right)\left(\begin{array}{l}
z_{1} \\
z_{2}
\end{array}\right)=\left(\begin{array}{c}
f_{1}(t) \\
0
\end{array}\right) \\
z_{1}(0)=z_{1}^{0}, \quad z_{2}(0)=z_{2}^{0}
\end{gathered}
$$

Здесь $z_{1} \in H_{1}$ - набор динамических переменных; $z_{2} \in H_{2}$ - набор кинематических переменных; $g>0$ - ускорение силы тяжести; $C_{1}$ - оператор кинетической энергии системы, $0 \ll C_{1} \in \mathcal{L}\left(H_{1}\right)$; $C_{2}$ - оператор потенциальной энергии, $C_{2}=C_{2}^{*} \in \mathcal{L}\left(H_{2}\right) ; 0 \leqslant A_{1}: \mathcal{D}\left(A_{1}\right) \subset H_{1} \rightarrow H_{1}-$ оператор диссипации энергии, а $B_{12}: \mathcal{D}\left(B_{12}\right) \subset H_{2} \rightarrow \mathcal{R}\left(B_{12}\right)=H_{1}$ и $B_{21}: \mathcal{D}\left(B_{21}\right) \subset H_{1} \rightarrow \mathcal{R}\left(B_{21}\right)=H_{2}-$ компоненты матричного антидиагонального оператора обмена энергиями (кинетической и потенциальной), $B_{12}^{*}=-B_{21} ; f_{1}(t)$ - заданное малое поле внешних сил.

Сильная разрешимость данной абстрактной задачи (для неограниченного оператора $A_{1}$, когда часть жидкостей являются вязкими) при выполнении описанных свойств операторных коэффициентов в случае $C_{2} \gg 0$ доказана в [15]. Если оператор $A_{1}$ является ограниченным, то разрешимость задачи (1) доказана в [16] как для положительно определенного, так и для не знакоопределенного оператора $C_{2}$. Сопутствующие спектральные задачи исследовались ранее либо для консервативных систем (когда все жидкости идеальные и трение в шарнирах не учитывается), либо для диссипативных систем (когда все жидкости вязкие и трение в шарнире учитывается). Изучение спектральных свойств частично диссипативной системы требует особого подхода. В данной работе рассмотрена базовая задача из этого класса, когда имеется одиночный маятник с трением в шарнире и полостью, частично заполненной одной идеальной жидкостью. 
2. Постановка задачи. Пусть имеется твердое тело $\Omega_{0}$ с плотностью $\rho_{0}$, которое закреплено с помощью сферического шарнира в неподвижной точке $O_{1}$, относительно которой тело совершает малые пространственные движения. Пусть внутри $\Omega_{0}$ имеется полость, частично заполненная однородной идеальной несжимаемой жидкостью с плотностью $\rho_{1}$, которая в состоянии равновесия под действием силы тяжести с ускорением $g$ занимает область $\Omega_{1}$, ограниченную твердой стенкой $S$ и горизонтальной равновесной свободной поверхностью $\Gamma$.

Введем подвижную (неинерциальную) систему декартовых координат $O_{1} x_{1}^{1} x_{2}^{1} x_{3}^{1}$ с ортами $\left\{\boldsymbol{e}_{1}^{k}\right\}_{k=1}^{3}$, жестко связанную с телом. Будем считать, что в состоянии равновесия сила тяжести противоположно направлена по отношению к $\boldsymbol{e}_{1}^{3}$, а плоскость $Г$ ортогональна этому вектору, при этом расстояние от точки подвеса до центра масс равно $l \geqslant 0$.

Из курса теоретической механики известно, что положение маятника можно однозначно описать с помощью вектора углового перемещения $\boldsymbol{\delta}(t)$, связанного с угловой скоростью $\boldsymbol{\omega}(t)$ с помощью связи $d \boldsymbol{\delta} d t=\boldsymbol{\omega}$. Движение жидкости в маятнике однозначно определяется полем относительных скоростей $\boldsymbol{u}(t, x)$, отклонением поля давлений от равновесного $p(t, x)$, а также малой функцией $\zeta(t, x), x \in \Gamma$, равной отклонению свободной поверхности жидкости от равновесного состояния вдоль нормали $\boldsymbol{n}$ к $Г$.

Уравнение изменения кинетического момента гидромеханической системы относительно точки $O_{1}$ после линеаризации (см. [17, с. 143-145]) приводит к соотношению

$$
\boldsymbol{J} \frac{d \omega}{d t}+\rho_{1} \int_{\Omega_{1}} \boldsymbol{r} \times \frac{\partial \boldsymbol{u}}{\partial t} d \Omega_{1}+\alpha \boldsymbol{\omega}+g m l P_{2} \boldsymbol{\delta}-g \rho_{1} \int_{\Gamma}\left(\boldsymbol{e}_{1}^{3} \times \boldsymbol{r}\right) \zeta d \Gamma=\boldsymbol{M}(t) .
$$

Здесь $\boldsymbol{r}$-радиус-вектор, идущий из точки подвеса $O_{1} ; m>0$-масса маятника с жидкостью; $\boldsymbol{M}(t)$ - заданный малый момент внешних сил; $\alpha \boldsymbol{\omega}(\alpha \geqslant 0)$ - момент сил трения в шарнире;

$$
P_{2} \boldsymbol{\delta}:=\sum_{j=1}^{2} \delta^{j} \boldsymbol{e}_{1}^{j}
$$

через

$$
\boldsymbol{J} \frac{d \boldsymbol{\omega}}{d t}:=\int_{\Omega_{0}} \boldsymbol{r} \times\left(\frac{d \boldsymbol{\omega}}{d t} \times \boldsymbol{r}\right) \rho_{0} d \Omega_{0}+\int_{\Omega_{1}} \boldsymbol{r} \times\left(\frac{d \boldsymbol{\omega}}{d t} \times \boldsymbol{r}\right) \rho_{1} d \Omega_{1}
$$

обозначен закон действия тензора инерции тела с затвердевшей жидкостью. Кроме этого, искомые переменные удовлетворяют линеаризованному уравнению Эйлера и уравнению неразрывности

$$
\rho_{1} \frac{\partial \boldsymbol{u}}{\partial t}+\rho_{1} \frac{d \boldsymbol{\omega}}{d t} \times \boldsymbol{r}+\nabla p=\boldsymbol{f}, \quad \operatorname{div} \boldsymbol{u}=0\left(\text { в } \Omega_{1}\right),
$$

краевым и начальным условиям (см. [17])

$$
\begin{gathered}
\boldsymbol{u} \cdot \boldsymbol{n}=0 \quad(\text { на } S), \\
\frac{\partial \zeta}{\partial t}=\boldsymbol{u} \cdot \boldsymbol{n}, \quad p=\rho_{1} g\left(\zeta+\theta\left(P_{2} \boldsymbol{\delta} \times \boldsymbol{r}\right) \cdot \boldsymbol{e}_{1}^{3}\right) \quad(\text { на } Г), \\
\boldsymbol{u}(0, x)=\boldsymbol{u}^{0}(x), \quad x \in \Omega_{1}, \quad \zeta(0, x)=\zeta^{0}(x), \quad x \in \Gamma, \\
\boldsymbol{\omega}(0)=\boldsymbol{\omega}^{0}, \quad \boldsymbol{\delta}(0)=\boldsymbol{\delta}^{0},
\end{gathered}
$$

где $\theta: L_{2}(\Gamma) \rightarrow L_{2, \Gamma}$ ортопроектор на подпространство $L_{2, \Gamma}:=L_{2}(\Gamma) \ominus \operatorname{sp}\left\{1_{\Gamma}\right\}$ функций, ортогональных константам на $\Gamma$, а также условию сохранения объема и дополнительным очевидным соотношениям

$$
\int_{\Gamma} \zeta d \Gamma=0, \quad \frac{d}{d t} P_{2} \boldsymbol{\delta}=P_{2} \boldsymbol{\omega}, \quad \frac{d}{d t} P^{3} \boldsymbol{\delta}=P^{3} \boldsymbol{\omega}, \quad P^{3}:=I-P_{2} .
$$


3. Применение операторного подхода. Теорема о сильной разрешимости. Задача (2)(8) исследуется методами функционального анализа. Будем считать, что поле скорости $\boldsymbol{u}$ является функцией $t$ со значениями в $\boldsymbol{L}_{2}\left(\Omega_{1}\right)$. Для данного пространства справедливо ортогональное разложение (см. $[17$, с. 106])

$$
\begin{aligned}
& \boldsymbol{L}_{2}\left(\Omega_{1}\right)=\boldsymbol{J}_{0}\left(\Omega_{1}\right) \oplus \boldsymbol{G}_{h, S}\left(\Omega_{1}\right) \oplus \boldsymbol{G}_{0, \Gamma}\left(\Omega_{1}\right), \\
& \left.\left.\boldsymbol{J}_{0}\left(\Omega_{1}\right):=\left\{\boldsymbol{u} \in \boldsymbol{L}_{2}\left(\Omega_{1}\right): \operatorname{div} \boldsymbol{u}=0 \text { (в } \Omega_{1}\right), \boldsymbol{u} \cdot \boldsymbol{n}=0 \text { (на } \partial \Omega_{1}\right)\right\}, \\
& \left.\left.\boldsymbol{G}_{h, S}\left(\Omega_{1}\right):=\left\{\nabla \Phi \in \boldsymbol{L}_{2}\left(\Omega_{1}\right): \Delta \Phi=0 \text { (в } \Omega_{1}\right), \frac{\partial \Phi}{\partial n}=0 \text { (на } S\right), \int_{\Gamma} \Phi d \Gamma=0\right\}, \\
& \left.\boldsymbol{G}_{0, \Gamma}\left(\Omega_{1}\right):=\left\{\nabla \varphi \in \boldsymbol{L}_{2}\left(\Omega_{1}\right): \varphi=0 \text { (на } \Gamma\right)\right\} .
\end{aligned}
$$

Подействуем на обе части уравнения (3) соответствующими ортопроекторами $P_{0}, P_{h, S}, P_{0, \Gamma}$, считая, что

$$
\boldsymbol{u}=\boldsymbol{w}+\nabla \Phi \in \boldsymbol{J}_{0}\left(\Omega_{1}\right) \oplus \boldsymbol{G}_{h, S}\left(\Omega_{1}\right), \quad \nabla p=\nabla \widetilde{p}+\nabla \varphi \in \boldsymbol{G}_{h, S}\left(\Omega_{1}\right) \oplus \boldsymbol{G}_{0, \Gamma}\left(\Omega_{1}\right) .
$$

Приходим к соотношениям

$$
\begin{gathered}
\rho_{1} \frac{\partial \boldsymbol{w}}{\partial t}+\rho_{1} P_{0}\left(\frac{d \boldsymbol{\omega}}{d t} \times \boldsymbol{r}\right)=P_{0} \boldsymbol{f} \\
\rho_{1} \frac{\partial}{\partial t} \nabla \Phi+\rho_{1} P_{h, S}\left(\frac{d \boldsymbol{\omega}}{d t} \times \boldsymbol{r}\right)+\nabla \widetilde{p}=P_{h, S} \boldsymbol{f} \\
\rho_{1} P_{0, \Gamma}\left(\frac{d \boldsymbol{\omega}}{d t} \times \boldsymbol{r}\right)+\nabla \varphi=P_{0, \Gamma} \boldsymbol{f} .
\end{gathered}
$$

Последнее уравнение является вспомогательным и позволяет найти неизвестное поле $\nabla \varphi$, если известны $\boldsymbol{f}$ и $\boldsymbol{\omega}$. Далее его не рассматриваем. При этом краевые условия (5) приводят к соотношениям

$$
\frac{\partial \zeta}{\partial t}=\gamma_{n}(\nabla \Phi):=\nabla \Phi \cdot \boldsymbol{n}=\frac{\partial \Phi}{\partial n}, \quad \widetilde{p}=\rho_{1} g\left(\zeta+\theta\left(P_{2} \boldsymbol{\delta} \times \boldsymbol{r}\right) \cdot \boldsymbol{e}_{1}^{3}\right) \quad(\text { на } \Gamma), \quad \int_{\Gamma} \widetilde{p} d \Gamma=0 .
$$

В силу условия $\nabla \widetilde{p} \in \boldsymbol{G}_{h, S}\left(\Omega_{1}\right)$ для нахождения потенциала $\widetilde{p}$ возникает краевая задача (Зарембы)

$$
\Delta \widetilde{p}=0\left(\text { в } \Omega_{1}\right), \quad \frac{\partial \widetilde{p}}{\partial n}=0(\text { на } S), \quad \widetilde{p}=\rho_{1} g\left(\zeta+\theta\left(P_{2} \boldsymbol{\delta} \times \boldsymbol{r}\right) \cdot \boldsymbol{e}_{1}^{3}\right)=: \psi(\text { на } \Gamma) .
$$

Известно (см. [17, с. 45-46]), что для области $\Omega_{1}$ с липшицевой границей такая задача имеет единственное слабое решение $\nabla \widetilde{p}=Q \psi \in \boldsymbol{G}_{h, S}\left(\Omega_{1}\right)$, если выполнено условие

$$
\psi \in H_{\Gamma}^{1 / 2}:=H^{1 / 2}(\Gamma) \cap L_{2, \Gamma} .
$$

Лемма 1. Операторы $Q: \mathcal{D}(Q)=H_{\Gamma}^{1 / 2}:=H^{1 / 2}(\Gamma) \cap L_{2, \Gamma} \subset L_{2, \Gamma} \rightarrow \boldsymbol{G}_{h, S}\left(\Omega_{1}\right) u$

$$
\gamma_{n}: \mathcal{D}\left(\gamma_{n}\right)=\left\{\nabla \Phi \in \boldsymbol{G}_{h, S}\left(\Omega_{1}\right): \frac{\partial \Phi}{\partial n} \in L_{2, \Gamma}\right\} \subset \boldsymbol{G}_{h, S}\left(\Omega_{1}\right) \rightarrow L_{2, \Gamma}
$$

являются взаимно сопряљсенными и ограниченными.

Доказательство можно найти в [17, с. 137-138], а также [3, с. 43-44].

Отсюда из (14) получаем уравнение

$$
\rho_{1} \frac{\partial}{\partial t} \nabla \Phi+\rho_{1} P_{h, S}\left(\frac{d \boldsymbol{\omega}}{d t} \times \boldsymbol{r}\right)+\rho_{1} g Q\left(\zeta+\theta\left(P_{2} \boldsymbol{\delta} \times \boldsymbol{r}\right) \cdot \boldsymbol{e}_{1}^{3}\right)=P_{h, S} \boldsymbol{f} .
$$

Уравнения (13), (18) и (2) можно переписать в виде одного операторного соотношения

$$
C_{1} \frac{d z_{1}}{d t}+A_{1} z_{1}+B_{12} z_{2}=f_{1}(t)
$$

в пространстве динамических переменных

$$
H_{1}:=\boldsymbol{J}_{0}\left(\Omega_{1}\right) \oplus \boldsymbol{G}_{h, S}\left(\Omega_{1}\right) \oplus \mathbb{C}^{3},
$$


где $z_{1}:=(\boldsymbol{w} ; \nabla \Phi ; \boldsymbol{\omega})^{\tau}, z_{2}:=\left(\zeta ; P_{2} \boldsymbol{\delta}\right)^{\tau}$. При этом

$$
\begin{aligned}
& C_{1} z_{1}:=\left(\rho_{1} \boldsymbol{w}+\rho_{1} P_{0}(\boldsymbol{\omega} \times \boldsymbol{r}) ; \rho_{1} \nabla \Phi+\rho_{1} P_{h, S}(\boldsymbol{\omega} \times \boldsymbol{r}) ;\right. \\
& \left.\rho_{1} \int_{\Omega_{1}}(\boldsymbol{r} \times \boldsymbol{w}) d \Omega_{1}+\rho_{1} \int_{\Omega_{1}}(\boldsymbol{r} \times \nabla \Phi) d \Omega_{1}+\boldsymbol{J} \boldsymbol{\omega}\right)^{\tau}, \\
& B_{12} z_{2}:=\left(0 ; \rho_{1} Q\left(\zeta+\theta\left(P_{2} \boldsymbol{\delta} \times \boldsymbol{r}\right) \cdot \boldsymbol{e}_{1}^{3}\right) ;-\rho_{1} \int_{\Gamma}\left(\boldsymbol{e}_{1}^{3} \times \boldsymbol{r}\right) \zeta d \Gamma+m l P_{2} \boldsymbol{\delta}\right)^{\tau} \\
& A_{1} z_{1}:=(\mathbf{0} ; \mathbf{0} ; \alpha \boldsymbol{\omega})^{\tau}, \quad f_{1}(t):=\left(P_{0} \boldsymbol{f} ; P_{h, S} \boldsymbol{f} ; \boldsymbol{M}\right) .
\end{aligned}
$$

Далее, кинематические соотношения

$$
\frac{\partial \zeta}{\partial t}=\gamma_{n}(\nabla \Phi)(\text { на } \Gamma), \quad \frac{d}{d t} P_{2} \boldsymbol{\delta}-P_{2} \boldsymbol{\omega}=\mathbf{0}\left(\text { в } \mathbb{C}^{2}\right)
$$

можно преобразовать в операторное соотношение

в пространстве кинетических переменных

$$
g C_{2} \frac{d z_{2}}{d t}+g B_{21} z_{1}=0
$$

$$
H_{2}:=L_{2, \Gamma} \oplus \mathbb{C}^{2}
$$

где

$$
\begin{aligned}
C_{2} z_{2} & =\left(\rho_{1}\left(\zeta+\theta\left(P_{2} \boldsymbol{\delta} \times \boldsymbol{r}\right) \cdot \boldsymbol{e}_{1}^{3}\right) ;-\rho_{1} \int_{\Gamma}\left(\boldsymbol{e}_{1}^{3} \times \boldsymbol{r}\right) \zeta d \Gamma+m l P_{2} \boldsymbol{\delta}\right)^{\tau} ; \\
B_{21} z_{1} & =\left(-\rho_{1}\left(\gamma_{n} \nabla \Phi+\theta\left(P_{2} \boldsymbol{\omega} \times \boldsymbol{r}\right) \cdot \boldsymbol{e}_{1}^{3}\right) ; \rho_{1} \int_{\Gamma}\left(\boldsymbol{e}_{1}^{3} \times \boldsymbol{r}\right)\left(\gamma_{n} \nabla \Phi\right) d \Gamma-m l P_{2} \boldsymbol{\omega}\right)^{\tau} .
\end{aligned}
$$

В [14, с. 172-173] (см. также [3, с. 40-42]) доказаны следующие утверждения.

Лемма 2. Если выполнено условие

$$
\Delta_{2}:=\left(m l-\rho_{1} \alpha_{11}\right)\left(m l-\rho_{1} \alpha_{22}\right)-\rho_{1} \alpha_{12}^{2} \neq 0, \quad \alpha_{j k}:=\int_{\Gamma}\left(\theta x_{1}^{j}\right) x_{1}^{k} d \Gamma=\alpha_{k j},
$$

то кинематические условия (20) равносильны дифберенциальной связи (21). При этом оператор $C_{2}$ (потенииальной энергии) обладает свойствами: $C_{2}=C_{2}^{*} \in \mathcal{L}\left(H_{2}\right), C_{2}^{-1} \in \mathcal{L}\left(H_{2}\right)$, а его квадратичная форма имеет не более $\varkappa=2$ отрицательных квадратов. Будем далее всюду считать, что условие (22) выполнено.

Лемма 3. При выполнении условий

$$
\Delta_{1}:=m l-\rho_{1} \alpha_{11}>0, \quad \Delta_{2}>0
$$

оператор потенциальной энергии $C_{2}$ положительно определен.

Определение 1. Будем говорить, что состояние равновесия системы статически устойчиво по линейному приближению, если $C_{2} \gg 0$. В противном случае состояние равновесия будем называть статически неустойчивым.

В статье [14, с. 169-170, 177-178] доказаны также следующие утверждения.

Лемма 4. Оператор $C_{1}: H_{1} \rightarrow H_{1}$ является ограниченным самосопряженным положительно определенным оператором. Его квадратичная форма

$$
\left(C_{1} z_{1}, z_{1}\right)_{H_{1}}=\boldsymbol{J} \boldsymbol{\omega} \cdot \boldsymbol{\omega}+\rho_{1} \int_{\Omega_{1}}|\boldsymbol{u}+(\boldsymbol{\omega} \times \boldsymbol{r})|^{2} d \Omega_{1}
$$


равна удвоенной кинетической энергии системы (далее будем называть оператор $C_{1}$ оператором кинетической энергии).

Лемма 5. Oператоры $B_{12}: H_{2} \rightarrow H_{1} u B_{21}: H_{1} \rightarrow H_{2}$ (обмена между кинетической и потенииальной энергиями) являются неограниченными кососамосопряэенными, т.е.

$$
\left(B_{12} z_{2}, z_{1}\right)_{H_{1}}=-\left(B_{21} z_{1}, z_{2}\right)_{H_{2}}, \quad \forall z_{1} \in \mathcal{D}\left(B_{21}\right), z_{2} \in \mathcal{D}\left(B_{12}\right)
$$

где $\mathcal{D}\left(B_{12}\right)=\mathcal{D}(Q) \oplus \mathbb{C}^{2}, \mathcal{D}(Q)=H_{\Gamma}^{1 / 2}$

Таким образом, исходная начально-краевая задача (2)-(8) в случае общего положения (22) сводится к задаче Коши (1), а также тривиальной связи

$$
\frac{d}{d t} P^{3} \boldsymbol{\delta}=P^{3} \boldsymbol{\omega} .
$$

При этом операторные коэффициенты обладают сформулированными выше общими свойствами и имеют непосредственный физический смысл. На основе результатов статьи [16] (см. также [14]), где доказана разрешимость задачи с трением для двух сочлененных тел с полостями, частично заполненными идеальными жидкостями, сформулируем итоговую теорему о разрешимости начально-краевой задачи (2)-(8).

Определение 2. Будем говорить, что задача (2)-(8) имеет сильное по переменной $t$ решение на отрезке $[0 ; T]$, если выполнены следующие условия:

(1) $\boldsymbol{u}(t, x) \in C^{1}\left([0 ; T] ; \boldsymbol{J}_{0, S}\left(\Omega_{1}\right)\right), \nabla p(t, x) \in C^{1}\left([0 ; T] ; \boldsymbol{G}\left(\Omega_{1}\right)\right), \zeta\left(t, x_{1}, x_{2}\right) \in C^{1}\left([0 ; T] ; L_{2, \Gamma}\right)$, $\boldsymbol{\omega}(t) \in C^{1}\left([0 ; T] ; \mathbb{C}^{3}\right), \boldsymbol{\delta}(t) \in C^{2}\left([0 ; T] ; \mathbb{C}^{3}\right)$

(2) при любом $t \in[0 ; T]$ выполнено уравнение (2), где все слагаемые являются элементами $C\left([0 ; T] ; \mathbb{C}^{3}\right)$

(3) выполнены начальные и краевые условия, причем первое соотношение $(5)$ в $C^{1}\left([0 ; T] ; L_{2, \Gamma}\right)$, а второе в $C^{1}\left([0 ; T] ; H_{\Gamma}^{1 / 2}\right)$.

Теорема 1. Пусть в задаче (2)-(8) выполнены условия

$$
\begin{aligned}
\boldsymbol{u}^{0} \in \boldsymbol{J}_{0}\left(\Omega_{1}\right) \oplus \boldsymbol{G}_{h, S}\left(\Omega_{1}\right), \quad P_{h, S} \boldsymbol{u}^{0}=: \nabla \Phi^{0} \in \boldsymbol{G}_{h, S}\left(\Omega_{1}\right):\left.\frac{\partial \Phi}{\partial n}\right|_{\Gamma} \in L_{2, \Gamma} \\
\zeta^{0} \in H_{\Gamma}^{1 / 2}, \quad \boldsymbol{\omega}^{0} \in C^{3}, \quad \boldsymbol{\delta}^{0} \in C^{3}, \quad \boldsymbol{f}(t) \in C^{1}\left([0 ; T] ; \boldsymbol{L}_{2}\left(\Omega_{1}\right)\right), \quad \boldsymbol{M}(t) \in C^{1}\left([0 ; T] ; \mathbb{C}^{3}\right) .
\end{aligned}
$$

Тогда эта задача имеет единственное сильное решение на отрезке [0; T], для которого выполнен закон баланса полной энергии

$$
\begin{aligned}
& \frac{1}{2} \frac{d}{d t}\left[\boldsymbol{J}_{m} \boldsymbol{\omega} \cdot \boldsymbol{\omega}+\rho_{1} \int_{\Omega_{1}}|\boldsymbol{u}+(\boldsymbol{\omega} \times \boldsymbol{r})|^{2} d \Omega_{1}+g m l\left|P_{2} \boldsymbol{\delta}\right|^{2}+\right. \\
& \left.\quad+g \rho_{1} \int_{\Gamma}\left(\left|\zeta+\theta\left(P_{2} \boldsymbol{\delta} \times \boldsymbol{r}\right) \cdot \boldsymbol{e}_{1}^{3}\right|^{2}-\left|\theta\left(P_{2} \boldsymbol{\delta} \times \boldsymbol{r}\right) \cdot \boldsymbol{e}_{1}^{3}\right|^{2}\right) d \Gamma\right]=-\alpha|\boldsymbol{\omega}|^{2}+\rho_{1} \int_{\Omega_{1}} \boldsymbol{f} \cdot \boldsymbol{u} d \Omega_{1}+\boldsymbol{M} \cdot \boldsymbol{\omega}
\end{aligned}
$$

где все слагаемые являются непрерывными функииями времени.

4. Спектральные свойства задачи в отсутствие трения в шарнире. Рассмотрим решения однородной задачи $(1)$, зависящие от времени по закону $e^{-\lambda t}, \lambda \in \mathbb{C}$. Получаем спектральную задачу

$$
\begin{gathered}
-\lambda C_{1} z_{1}+A_{1} z_{1}+g B_{12} z_{2}=0\left(\text { в } H_{1}\right) \\
-\lambda C_{2} z_{1}+B_{21} z_{1}=0\left(\text { в } H_{2}\right) \\
-\lambda P^{3} \boldsymbol{\delta}=P^{3} \boldsymbol{\omega}(\text { в } \mathbb{C}) .
\end{gathered}
$$


Лемма 6. При $\lambda=0$ спектральная задача (25)-(27) имеет ненулевое решение вида

$$
z_{1}=\left(\boldsymbol{w}_{0} ; \mathbf{0} ; \mathbf{0}\right)^{\tau}, \quad \forall \boldsymbol{w}_{0} \in \boldsymbol{J}_{0}\left(\Omega_{1}\right), \quad z_{2}=(0 ; \mathbf{0})^{\tau}, \quad \forall P^{3} \boldsymbol{\delta} \neq 0,
$$

т.е. $\lambda=0$ является бесконечнократным собственным значением.

Доказательство. Согласно лемме 2 из условия $B_{21} z_{1}=0$ следует выполнение условий

Отсюда

$$
\gamma_{n}(\nabla \Phi)=0, \quad \frac{d}{d t} P_{2} \boldsymbol{\delta}=P_{2} \boldsymbol{\omega}
$$

Значит, в силу связи

$$
\nabla \Phi=0, \quad P_{2} \boldsymbol{\omega}=\mathbf{0}
$$

$$
-\lambda \frac{d}{d t} P^{3} \boldsymbol{\delta}=P^{3} \boldsymbol{\omega}
$$

имеем $\boldsymbol{\omega}=\mathbf{0}$. Далее, из условия $g B_{12} z_{2}+A_{1} z_{1}=0$ получаем, что $B_{12} z_{2}=0$, отсюда $\zeta=0, P_{2} \boldsymbol{\delta}=\mathbf{0}$. Непосредственной проверкой убеждаемся, что решения вида (28) удовлетворяют задаче (25)-(26) при $\lambda=0$.

Замечание. Решениям вида (28) соответствуют стационарные движения жидкости, при которых свободная поверхность Г остается неподвижной. При этом маятник с полостью не покачивается, но может быть повернут на произвольный угол $\delta^{3}$ вдоль оси $\boldsymbol{e}_{1}$, что соответствует переходу в новое состояние равновесия.

Рассмотрим сейчас свойства задачи (25)-(26) при $\lambda \neq 0$ в случае, когда трение в шарнирах не учитывается, т.е. когда $\alpha=0$, а значит, $A_{1}=0$. Перейдем от уравнения $(25)$ к соответствующей системе

$$
\begin{aligned}
& -\lambda \rho_{1}\left(\boldsymbol{w}+P_{0}(\boldsymbol{\omega} \times \boldsymbol{r})\right)=\mathbf{0} \\
& -\lambda \rho_{1}\left(\nabla \Phi+P_{h, S}(\boldsymbol{\omega} \times \boldsymbol{r})\right)+g \rho_{1} Q\left(\zeta+\theta\left(P_{2} \boldsymbol{\delta} \times \boldsymbol{r}\right) \cdot \boldsymbol{e}_{1}^{3}\right)=\mathbf{0} \\
& -\lambda\left[\rho_{1} \int_{\Omega_{1}}(\boldsymbol{r} \times \boldsymbol{w}) d \Omega_{1}+\rho_{1} \int_{\Omega_{1}}(\boldsymbol{r} \times \nabla \Phi) d \Omega_{1}+\boldsymbol{J} \boldsymbol{\omega}\right]+g\left[-\rho_{1} \int_{\Gamma}\left(\boldsymbol{e}_{1}^{3} \times \boldsymbol{r}\right) \zeta d \Gamma+m l P_{2} \boldsymbol{\delta}\right]=\mathbf{0} .
\end{aligned}
$$

Из (29) получаем, что

$$
\boldsymbol{w}=-P_{0}(\boldsymbol{\omega} \times \boldsymbol{r})
$$

Отсюда в третьем уравнении выполнено соотношение

$$
\begin{array}{r}
\rho_{1} \int_{\Omega_{1}}(\boldsymbol{r} \times \boldsymbol{w}) d \Omega_{1}+\boldsymbol{J} \boldsymbol{\omega}=\rho_{0} \int_{\Omega_{0}} \boldsymbol{r} \times(\boldsymbol{\omega} \times \boldsymbol{r}) d \Omega_{0}+\rho_{1} \int_{\Omega_{1}} \boldsymbol{r} \times\left(\left(I-P_{0}\right)(\boldsymbol{\omega} \times \boldsymbol{r}) d \Omega_{1}=:\right. \\
=: \boldsymbol{J}_{\mathrm{T}} \boldsymbol{\omega}+\boldsymbol{J}_{\text {пр, жж},} \boldsymbol{\omega},
\end{array}
$$

где $\boldsymbol{J}_{\mathrm{T}}$ - тензор инерции твердой части маятника, а $\boldsymbol{J}_{\text {пр, ж }}$-так называемый присоединенный тензор инерции жидкости.

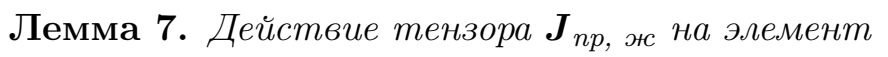

$$
\boldsymbol{\omega}=\sum_{k=1}^{3} \omega^{k} \boldsymbol{e}_{1}^{k}
$$

выражсается по формуле

$$
\boldsymbol{J}_{n p, \varkappa} \boldsymbol{\omega}=\rho_{1} \int_{\Omega_{1}} \boldsymbol{r} \times\left(\left(I-P_{0}\right)(\boldsymbol{\omega} \times \boldsymbol{r})\right) d \Omega_{1}=\rho_{1} \int_{\Omega_{1}} \boldsymbol{r} \times \nabla \psi d \Omega_{1},
$$

əde

$$
\nabla \psi=\sum_{k=1}^{3} \omega^{k} \nabla \psi_{k}
$$


а функции $\left\{\psi_{k}\right\}_{k=1}^{3}$ (потенциаль Жуковского) зависят лишь от формы области $\Omega_{1}$ и являются решениями краевой задачи

$$
\Delta \psi_{k}=0\left(\text { в } \Omega_{1}\right), \quad \frac{\partial \psi_{k}}{\partial n}=\left(\boldsymbol{e}_{1}^{k} \times \boldsymbol{r}\right) \cdot \boldsymbol{n}\left(\text { на } \partial \Omega_{1}\right), \quad k=1,2,3 .
$$

Доказательство. Действительно, в силу выполнения свойства $\operatorname{div}(\boldsymbol{\omega} \times \boldsymbol{r})=0$ поле $\left(I-P_{0}\right)(\boldsymbol{\omega} \times \boldsymbol{r})$ является потенциально-гармоническим, а значит, $\boldsymbol{\omega} \times \boldsymbol{r}=\nabla \psi$, где $\Delta \psi=0$ (в $\Omega_{1}$ ).

С другой стороны, если $\psi_{k}$ являются решениями задачи (34), то функция

$$
\varphi=\sum_{k=1}^{3} \omega^{k} \psi_{k}
$$

является гармонической в области $\Omega_{1}$, а на границе области выполнено свойство

$$
\frac{\partial \varphi}{\partial n}=\left(\sum_{k=1}^{3} \omega^{k} \boldsymbol{e}_{1}^{k} \times \boldsymbol{r}\right) \cdot \boldsymbol{n}=(\boldsymbol{\omega} \times \boldsymbol{r}) \cdot \boldsymbol{n}=\frac{\partial \psi}{\partial n} .
$$

Так как гармоническая функция однозначно определяется своим значением на границе, то $\varphi=\psi$.

Уравнения (30), (31) переписываются в виде операторного соотношения

$$
-\lambda \widetilde{C}_{1} \widetilde{z}_{1}+g \widetilde{Q} C_{2} z_{2}=0
$$

где $\widetilde{z}_{1}:=(\nabla \Phi ; \boldsymbol{\omega})^{\tau}$ - элемент пространства

$$
\begin{gathered}
\widetilde{H}_{1}:=\boldsymbol{G}_{h, S}\left(\Omega_{1}\right) \oplus \mathbb{C}^{3}, \\
\widetilde{C}_{1} \widetilde{z}_{1}:=\left(\rho_{1}\left(\nabla \Phi+P_{h, S}(\boldsymbol{\omega} \times \boldsymbol{r})\right) ; \rho_{1} \int_{\Omega_{1}}(\boldsymbol{r} \times \nabla \Phi) d \Omega_{1}+\left(\boldsymbol{J}_{\mathrm{T}}+\boldsymbol{J}_{\mathrm{пр}, ~}\right) \boldsymbol{\omega}\right)^{\tau}, \quad \widetilde{Q}:=\operatorname{diag}\left(Q ; P_{2}\right) .
\end{gathered}
$$

Лемма 8. Оператор $\widetilde{C}_{1}$ обладает свойствами $0 \ll \widetilde{C}_{1} \in \mathcal{L}\left(\widetilde{H}_{1}\right)$, при этом

$$
\left(\widetilde{C}_{1} \widetilde{z}_{1}, \widetilde{z}_{1}\right)_{\widetilde{H}_{1}}=\left(\boldsymbol{J}_{m} \boldsymbol{\omega}\right) \cdot \boldsymbol{\omega}+\rho_{1} \int_{\Omega_{1}}\left|\nabla \Phi+\sum_{k=1}^{3} \omega^{k} \nabla \psi_{k}\right|^{2} d \Omega_{1} .
$$

Доказательство. С помощью непосредственного подсчета квадратичных форм можно проверить, что $\left(C_{1} z_{1}, z_{1}\right)_{H_{1}}=\left(\widetilde{C}_{1} \widetilde{z}_{1}, \widetilde{z}_{1}\right)_{\widetilde{H}_{1}}$ для произвольного элемента $z_{1}=(\boldsymbol{w} ; \nabla \Phi ; \boldsymbol{\omega})^{\tau}$, если $\boldsymbol{w}$ и $\boldsymbol{\omega}$ связаны соотношением (32). Отсюда, используя лемму 4 , получаем

$$
\left(\widetilde{C}_{1} \widetilde{z}_{1}, \widetilde{z}_{1}\right)_{\widetilde{H}_{1}} \geqslant c_{1}\left\|z_{1}\right\|_{H_{1}}^{2} \geqslant c_{1}\left\|\widetilde{z}_{1}\right\|_{\widetilde{H}_{1}}^{2}, \quad c_{1}>0 .
$$

Доказательство формулы (36) получаем, используя формулу (33). Действительно, с учетом соотношения

получаем

$$
\nabla \psi=\boldsymbol{\omega} \times \boldsymbol{r}=\sum_{k=1}^{3} \omega^{k} \nabla \psi_{k}
$$

$$
\begin{array}{r}
\left(\widetilde{C}_{1} \widetilde{z}_{1}, \widetilde{z}_{1}\right)_{\widetilde{H}_{1}}=\rho_{1} \int_{\Omega_{1}}|\nabla \Phi|^{2} d \Omega_{1}+2 \rho_{1} \int_{\Omega_{1}} \nabla \Phi \cdot(\boldsymbol{\omega} \times \boldsymbol{r}) d \Omega_{1}+\left(\boldsymbol{J}_{\mathrm{T}} \boldsymbol{\omega}\right) \cdot \boldsymbol{\omega}+\left(\boldsymbol{J}_{\text {пр, ж}} \boldsymbol{\omega}\right) \cdot \boldsymbol{\omega}= \\
=\left(\boldsymbol{J}_{\mathrm{T}} \boldsymbol{\omega}\right) \cdot \boldsymbol{\omega}+\rho_{1} \int_{\Omega_{1}}|\nabla \Phi|^{2} d \Omega_{1}+2 \rho_{1} \int_{\Omega_{1}} \nabla \Phi \cdot \nabla \psi d \Omega_{1}+\rho_{1} \int_{\Omega_{1}}|\nabla \psi|^{2} d \Omega_{1}= \\
=\left(\boldsymbol{J}_{\mathrm{T}} \boldsymbol{\omega}\right) \cdot \boldsymbol{\omega}+\rho_{1} \int_{\Omega_{1}}|\nabla \Phi+\nabla \psi|^{2} d \Omega_{1} .
\end{array}
$$


В силу леммы 2 уравнение (26) равносильно соотношениям

$$
-\lambda \zeta=\gamma_{n} \nabla \Phi\left(\text { в } L_{2, \Gamma}\right), \quad-\lambda P_{2} \boldsymbol{\delta}=P_{2} \boldsymbol{\omega}\left(\text { в } \mathbb{C}^{2}\right) .
$$

Используя оператор $\widetilde{\gamma}_{n}=\operatorname{diag}\left(\gamma_{n} ; P_{2}\right)$, перепишем их в виде одного уравнения

$$
-\lambda z_{2}=\widetilde{\gamma}_{n} \widetilde{z}_{1}\left(\text { в } H_{2}\right) .
$$

Отсюда для $\lambda \neq 0$ имеем $z_{2}=-\lambda^{-1} \widetilde{\gamma}_{n} \widetilde{z}_{1}$, а значит, уравнение $(25)$ сводится к спектральной задаче

$$
g \widetilde{Q} C_{2} \widetilde{\gamma}_{n} \widetilde{z}_{1}=\mu \widetilde{C}_{1} \widetilde{z}_{1}\left(\text { в } \widetilde{H}_{1}\right), \quad \mu:=-\lambda^{2} .
$$

Лемма 9. Задача (37) имеет однократное собственное значение $\mu=0$, которому отвечает собственная функиия $\widetilde{z}_{1}^{0}=\left(\mathbf{0} ; P^{3} \boldsymbol{\omega}\right)^{\tau} \in \widetilde{H}_{1,0}, \forall P^{3} \boldsymbol{\omega} \in \mathbb{C}$.

Доказательство. Так как согласно лемме 2 оператор $C_{2}$ обратим, а оператор $\widetilde{Q}: H_{2} \rightarrow \widetilde{H}_{1}$ имеет нулевое ядро, то при $\mu=0$ справедливо соотношение

$$
\widetilde{\gamma}_{n} \widetilde{z}_{1}=\left(\left.\frac{\partial \Phi}{\partial n}\right|_{\Gamma} ; P_{2} \boldsymbol{\omega}\right)^{\tau}=\mathbf{0}
$$

Отсюда в силу условия $\nabla \Phi \in \boldsymbol{G}_{h, S}\left(\Omega_{1}\right)$ получаем, что $\Phi=0, P_{2} \boldsymbol{\omega}=\mathbf{0}$, при этом третья компонента $P^{3} \boldsymbol{\omega}=\boldsymbol{\omega} \cdot \boldsymbol{e}_{1}^{3}$ может быть любой.

Лемма 10. В случае статической устойчивости, m.е. при $C_{2} \gg 0$, задача (37) на подпространстве $\widetilde{H}_{1,1}:=\widetilde{H}_{1} \ominus \widetilde{H}_{1,0}=\boldsymbol{G}_{h, S}\left(\Omega_{1}\right) \oplus \mathbb{C}^{2}$ имеет дискретный положительный спектр $\left\{\mu_{k}\right\}_{k=1}^{\infty}$ с единственной предельной точкой $+\infty$. При этом собственные значения $\mu_{k}$ являются последовательными минимумами вариационного отношения

$$
\frac{g\left(C_{2} \widetilde{\gamma}_{n} \widetilde{z}_{1}, \widetilde{\gamma}_{n} \widetilde{z}_{1}\right)_{H_{2}}}{\left(\widetilde{C}_{1} \widetilde{z}_{1}, \widetilde{z}_{1}\right)_{H_{1}}}
$$

на элементах $\widetilde{z}_{1}=\left(\nabla \Phi ; P_{2} \boldsymbol{\omega}+P^{3} \boldsymbol{\omega}\right)^{\tau} \in \widetilde{H}_{1}$, где $P^{3} \boldsymbol{\omega}$ однозначно определяется по $\nabla \Phi$ и $P_{2} \boldsymbol{\omega}:$

$$
\begin{gathered}
P^{3} \boldsymbol{\omega}=-\beta^{-1}\left[\rho_{1} P^{3} \int_{\Omega_{1}}\left(\boldsymbol{r}_{1} \times \nabla \Phi\right) d \Omega_{1}+\left(P^{3} \boldsymbol{J}_{1} P_{2}\right)\left(P_{2} \boldsymbol{\omega}\right)\right], \\
\boldsymbol{J}_{1}:=\boldsymbol{J}_{m}+\boldsymbol{J}_{n p, \varkappa}, \quad \beta:=P^{3} \boldsymbol{J}_{1} P^{3}>0 .
\end{gathered}
$$

Доказательство. В самом деле, операторы

$$
\begin{gathered}
\widetilde{Q}=\operatorname{diag}\left(Q ; P_{2}\right): H_{\Gamma}^{1 / 2} \oplus \mathbb{C}^{2} \rightarrow \boldsymbol{G}_{h, S}\left(\Omega_{1}\right) \oplus \mathbb{C}^{2}, \\
\widetilde{\gamma}_{n}=\operatorname{diag}\left(\gamma_{n} ; P_{2}\right): \mathcal{D}\left(\gamma_{n}\right) \oplus \mathbb{C}^{2} \rightarrow L_{2, \Gamma} \oplus \mathbb{C}^{2}
\end{gathered}
$$

являются ограниченными и ограниченно обратимыми, поэтому

$$
\begin{gathered}
\widetilde{Q}^{-1}=\operatorname{diag}\left(Q^{-1} ; P_{2}\right) \in \mathcal{L}\left(\boldsymbol{G}_{h, S}\left(\Omega_{1}\right) \oplus \mathbb{C}^{2} ; H_{\Gamma}^{1 / 2} \oplus \mathbb{C}^{2}\right), \\
\widetilde{\gamma}_{n}^{-1}=\operatorname{diag}\left(\gamma_{n}^{-1} ; P_{2}\right) \in \mathcal{L}\left(L_{2, \Gamma} \oplus \mathbb{C}^{2} ; \mathcal{D}\left(\gamma_{n}\right) \oplus \mathbb{C}^{2}\right) .
\end{gathered}
$$

Осуществим в задаче (37) замену переменной $\widehat{z}_{1}:=\left(\varphi ; P_{2} \boldsymbol{\omega}\right)^{\tau} \in L_{2, \Gamma} \oplus \mathbb{C}^{2}=H_{2}$, где элемент $\varphi:=\gamma_{n} \nabla \Phi$, тогда $\widetilde{z}_{1}=(\nabla \Phi ; \boldsymbol{\omega})^{\tau}=\left(\gamma_{n}^{-1} \varphi ; P_{2} \boldsymbol{\omega}\right)^{\tau}=\widetilde{\gamma}_{n}^{-1} \widehat{z}_{1} \in \widetilde{H}_{1,1}$. Отсюда задача $(37)$ сводится к задаче

$$
g C_{2} \widehat{z}_{1}=\mu \widetilde{Q}^{-1} \widetilde{C}_{1} \widetilde{\gamma}_{n}^{-1} \widehat{z}_{1}=: \mu \widehat{C}_{1} \widehat{z}_{1}\left(\text { в } H_{2}\right) .
$$

Здесь вместо $\widetilde{C}_{1}$ понимается его сужение $\widehat{C}_{1}$ на подпространство $\widetilde{H}_{1,1}$. Оператор $\widehat{C}_{1}$ остается положительным ограниченным оператором. В силу того, что $\mathcal{D}\left(\gamma_{n}\right) \subset \boldsymbol{G}_{h, S}\left(\Omega_{1}\right)$, а $H_{\Gamma}^{1 / 2}$ компактно вложено в $L_{2, \Gamma}$, оператор $\widehat{C}_{1}$ является компактным и положительным в $H_{2}$. 
Так как оператор $C_{2}$ является ограниченным и положительно определенным, то задача (37) сводится к нахождению характеристических чисел компактного положительного оператора. Отсюда из теоремы Гильберта-Шмидта следует, что спектр задачи (40) является дискретным и положительным. При этом система соответствующих собственных элементов $\left\{\widehat{z}_{1, k}\right\}_{k=1}^{\infty}$ образует базис в $H_{2}$, ортогональный по формам операторов $C_{2}$ и $\widehat{C}_{1}$ :

$$
\left(\widehat{C}_{1} \widehat{z}_{1, k}, \widehat{z}_{1, l}\right)_{H_{2}}=\delta_{k l}, \quad g\left(C_{2} \widehat{z}_{1, k}, \widehat{z}_{1, l}\right)_{H_{2}}=\mu_{k} \delta_{k l},
$$

а собственные значения $\mu_{k}$ являются последовательными минимумами вариационного отношения

$$
\frac{g\left(C_{2} \widehat{z}_{1}, \widehat{z}_{1}\right)_{H_{2}}}{\left(\widehat{C}_{1} \widehat{z}_{1}, \widehat{z}_{1}\right)_{H_{2}}} .
$$

Возвращаясь по замене $\widehat{z}_{1}=\widetilde{\gamma}_{n} \widetilde{z}_{1}$ к переменной $\widetilde{z}_{1}$, получаем вариационное отношение (38) с неопределенной компонентой $P^{3} \omega$ поля $\boldsymbol{\omega} \in \mathbb{C}^{3}$.

Для определения этой компоненты необходимо спроектировать (37) на подпространство $\widetilde{H}_{1,0}$, вспоминая действие оператора $\widetilde{C}_{1}$. Используя ортопроектор $\operatorname{diag}\left(0 ; P^{3}\right)$ в разложении

$$
\widetilde{H}_{1}=\left(\boldsymbol{G}_{h, S}\left(\Omega_{1}\right) \oplus \mathbb{C}^{2}\right) \oplus \mathbb{C}=\widetilde{H}_{1,1} \oplus \widetilde{H}_{1,0},
$$

получаем соотношение

$$
\rho_{1} P^{3} \int_{\Omega_{1}}(\boldsymbol{r} \times \nabla \Phi) d \Omega_{1}+P^{3}\left(\boldsymbol{J}_{\mathrm{T}}+\boldsymbol{J}_{\text {пр, ж}}\right)\left(P_{2} \boldsymbol{\omega}+P^{3} \boldsymbol{\omega}\right)=\mathbf{0},
$$

из которого следует формула (39).

Непосредственный подсчет приводит к формуле

$$
\left(C_{2} \widetilde{\gamma}_{n} \widetilde{z}_{1}, \widetilde{\gamma}_{n} \widetilde{z}_{1}\right)_{H_{2}}=\rho_{1} \int_{\Gamma}\left|\frac{\partial \Phi}{\partial n}+\theta\left(\left(P_{2} \boldsymbol{\omega} \times \boldsymbol{r}\right) \cdot \boldsymbol{e}_{1}^{3}\right)\right|^{2} d \Gamma-\rho_{1} \int_{\Gamma}\left|\left(P_{2} \boldsymbol{\omega} \times \boldsymbol{r}\right) \cdot \boldsymbol{e}_{1}^{3}\right|^{2} d \Gamma+m l\left|P_{2} \boldsymbol{\omega}\right|^{2} .
$$

Отсюда с учетом лемм 8, 10 получаем следующее утверждение.

Лемма 11. Положительные собственные значения задачи (37) находятся как последовательные минимумы вариационного отношения (38), равного выражснию

$$
g \frac{\rho_{1} \int_{\Gamma}\left|\frac{\partial \Phi}{\partial n}+\theta\left(\left(P_{2} \boldsymbol{\omega} \times \boldsymbol{r}\right) \cdot \boldsymbol{e}_{1}^{3}\right)\right|^{2} d \Gamma-\rho_{1} \int_{\Gamma}\left|\left(P_{2} \boldsymbol{\omega} \times \boldsymbol{r}\right) \cdot \boldsymbol{e}_{1}^{3}\right|^{2} d \Gamma+m l\left|P_{2} \boldsymbol{\omega}\right|^{2}}{\left(\boldsymbol{J}_{m} \boldsymbol{\omega}\right) \cdot \boldsymbol{\omega}+\rho_{1} \int_{\Omega_{1}}\left|\nabla \Phi+\sum_{k=1}^{3} \omega_{k} \nabla \psi_{k}\right|^{2} d \Omega_{1}},
$$

на элементах $\nabla \Phi \in \boldsymbol{G}_{h, S}\left(\Omega_{1}\right)$, обладающих свойствами

$$
\Delta \Phi=0\left(\text { в } \Omega_{1}\right), \quad \frac{\partial \Phi}{\partial n}=0(\text { на } S), \quad \int_{\Gamma} \Phi d \Gamma=0,
$$

и на векторе

$$
\boldsymbol{\omega}=\sum_{k=1}^{3} \omega^{k} \boldsymbol{e}_{1}^{k} \in \mathbb{C}^{3}
$$

где третъя компонента $\omega^{3}$ находится через

$$
P_{2} \boldsymbol{\omega}=\sum_{k=1}^{2} \omega^{k} \boldsymbol{e}_{1}^{k}
$$

$u \nabla \Phi$ по формуле (39). 
Замечание. Согласно общим результатам М. Ш. Бирмана и М. З. Соломяка (см., например, [6]) асимптотика вариационного отношения (42) аналогична асимптотике вариационного отношения «невозмущенной задачи» (соответствующей проблеме малых колебаний идеальной жидкости в неподвижном сосуде)

$$
g \int_{\Gamma}\left|\frac{\partial \Phi}{\partial n}\right|^{2} d \Gamma / \int_{\Omega_{1}}|\nabla \Phi|^{2} d \Omega_{1} .
$$

Отсюда согласно результатам И. Л. Вулис и М. З. Соломяка (см. $[9,10])$ следует асимптотика собственных значений задачи (37):

$$
\mu_{k}=g\left(\frac{|\Gamma|}{4 \pi}\right)^{-1 / 2} k^{1 / 2}[1+o(1)], \quad k \rightarrow \infty .
$$

На основании сформулированных выше лемм получаем следующее утверждение о свойствах исходной спектральной задачи (25)-(27), а также задачи (37).

Теорема 2. В случае статической устойчивости по линейному приближению (т.е. при выполнении условий (23)) в отсутствие трения в шаринире (т.е. при $\alpha=0$ ) задача (25)-(27) имеет ненулевой дискретный спектр $\left\{\lambda_{k}^{ \pm}\right\}_{k=1}^{\infty}$, расположенный комплексно сопряженными парами на мнимой оси. При этом имеет место асимптотика

$$
\lambda_{k}^{ \pm}= \pm i \mu_{k}^{1 / 2}= \pm i \sqrt{g}\left(\frac{|\Gamma|}{4 \pi}\right)^{-1 / 4} k^{1 / 4}[1+o(1)], \quad k \rightarrow \infty .
$$

Система соответствующих собственных элементов $\left(\widetilde{z}_{1}\right)_{j}=\left(\nabla \Phi ; P_{2} \boldsymbol{\omega}\right)_{j}$ образует ортогональный базис в $\widetilde{H}_{1,1}$ по квадратичным формам отношения (38):

$$
\left(\widetilde{C}_{1}\left(\widetilde{z}_{1}\right)_{j},\left(\widetilde{z}_{1}\right)_{k}\right)_{\widetilde{H}_{1}}=\delta_{j k}, \quad g\left(C_{2} \widetilde{\gamma}_{n}\left(\widetilde{z}_{1}\right)_{j}, \widetilde{\gamma}_{n}\left(\widetilde{z}_{1}\right)_{k}\right)_{H_{2}}=\mu_{k} \delta_{j k} .
$$

В случае отсутствия статической устойчивости оператор $C_{2}$ может иметь не более $\varkappa=2$ отрицательных собственных значений. Поскольку он ограничен и ограниченно обратим, то

$$
C_{2}=J_{\varkappa}\left|C_{2}\right|=\left|C_{2}\right|^{1 / 2} J_{\varkappa}\left|C_{2}\right|^{1 / 2},
$$

где

$$
0 \ll\left|C_{2}\right| \in \mathcal{L}\left(H_{2}\right), \quad J_{\varkappa}=J_{\varkappa}^{-1}=J_{\varkappa}^{*} .
$$

При этом спектральная задача (37) после замены $v:=\left|C_{2}\right|^{1 / 2} \widetilde{\gamma}_{n} \widetilde{z}_{1}$ сводится (аналогично (40)) к проблеме

$$
g v=\mu J_{\varkappa}\left|C_{2}\right|^{-1 / 2} \widetilde{Q}^{-1} \widetilde{C}_{1} \widetilde{\gamma}_{n}^{-1}\left|C_{2}\right|^{-1 / 2} v=: \mu J_{\varkappa} C v\left(\text { в } H_{2}\right),
$$

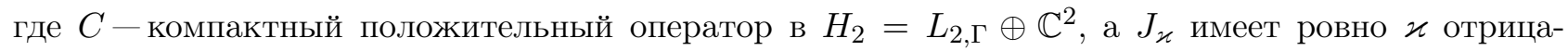
тельных собственных значений. Оператор $J_{\varkappa} C$ является компактным $J$-положительным в пространстве Понтрягина $\Pi_{\varkappa}=H_{2}$ с индефинитным скалярным произведением $[u, v]_{\Pi_{\varkappa}}:=\left(J_{\varkappa} u, v\right)_{H_{2}}$. Отсюда по теореме Л. С. Понтрягина (см. $[2,31])$ получаем, что задача (45) имеет ровно $\varkappa$ отрицательных собственных значений, при этом остальные положительны и сходятся к $+\infty$ (асимптотика (43) сохраняется). Соответствующая система собственных элементов образует базис Рисса в $H_{2}$, который является $J$-ортогональным:

$$
\left(C v_{j}, v_{k}\right)_{H_{2}}=\delta_{j k}, \quad g\left[v_{j}, v_{k}\right]_{\Pi_{\varkappa}}=\mu_{k} \delta_{j k} .
$$

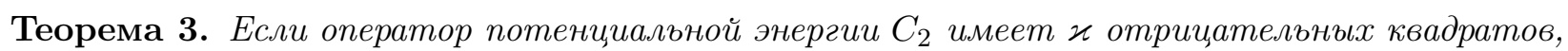
$1 \leqslant \varkappa \leqslant 2$, то задача (25)-(27) помимо бесконечнократного нулевого собственного значе-

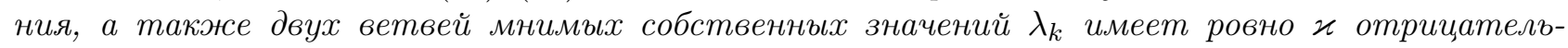
ных собственных значений, при этом соответствующая система собственных элементов $\left(\left.(\partial \Phi / \partial n)\right|_{\Gamma} ; P_{2} \boldsymbol{\omega}\right)$ образует базис Рисса в $H_{2}$. 
Замечание. Следствием данной теоремы является утверждение, которое в теоретической механике называют обращением теоремы Лагранжа об устойчивости. А именно, если система не является статически устойчивой по линейному приближению, т.е. условия (23) не выполнены, то система является также динамически неустойчивой, т.е. имеются решения начально-краевой задачи (2)-(8), экспоненциально возрастающие при $t \rightarrow+\infty$.

5. Локализация собственных значений и базисность системы корневых элементов при учете сил трения в шарнире. Вернемся к спектральной задаче (25)-(35) при условии $\alpha>0$. Здесь для $\lambda \neq 0$ из уравнения (25) вновь возникает связь $\boldsymbol{w}=-P_{0}(\boldsymbol{\omega} \times \boldsymbol{r})$, также выполнено уравнение (30), а в левой части (31) нужно добавить дополнительное слагаемое $\alpha \boldsymbol{\omega}$. Отсюда для элемента $\widetilde{z}_{1}=(\nabla \Phi ; \boldsymbol{\omega})^{\tau} \in \widetilde{H}_{1}=\boldsymbol{G}_{h, S}\left(\Omega_{1}\right) \oplus \mathbb{C}^{3}$ возникает соотношение

$$
-\lambda \widetilde{C}_{1} \widetilde{z}_{1}+\alpha P \widetilde{z}_{1}+g \widetilde{Q} C_{2} z_{2}=0
$$

где $P:=\operatorname{diag}\left(0 ; I_{3}\right), I_{3}$ - единичный оператор в $\mathbb{C}^{3}$. Отсюда с учетом связи

$$
-\lambda z_{2}-\widetilde{\gamma}_{n} \widetilde{z}_{1}=0
$$

взамен (37) возникает задача на собственные значения самосопряженного операторного пучка

$$
L_{\alpha}(\lambda) \widetilde{z}_{1}:=\left(\widetilde{C}_{2}-\lambda(\alpha P)+\lambda^{2} \widetilde{C}_{1}\right) \widetilde{z}_{1}=0, \quad \widetilde{C}_{2}:=g \widetilde{Q} C_{2} \widetilde{\gamma}_{n},
$$

с неограниченным операторным коэффициентом $\widetilde{C}_{2}$. Аналогично предыдущему пункту эта задача имеет однократное нулевое собственное значение, которому соответствует собственная функция $\widetilde{z}_{1}^{0}=\left(\mathbf{0} ; P^{3} \boldsymbol{\omega}\right)^{\tau} \in \widetilde{H}_{1,0}, \forall P^{3} \boldsymbol{\omega} \in \mathbb{C}$. При этом ненулевым собственным значениям отвечают собственные функции $(\nabla \Phi ; \boldsymbol{\omega})^{\tau}$, для которых компонента $P^{3} \boldsymbol{\omega}$ однозначно определяется по $\nabla \Phi \in$ $\boldsymbol{G}_{h, S}\left(\Omega_{1}\right)$ и $P_{2} \boldsymbol{\omega} \in \mathbb{C}^{2}$. Рассмотрим далее пучок $L_{\alpha}(\lambda)$ на подпространстве $\widetilde{H}_{1,1}=\boldsymbol{G}_{h, S}\left(\Omega_{1}\right) \oplus \mathbb{C}^{2}$, которое для простоты будем вновь обозначать $\widetilde{H}_{1}$.

Отметим, что на $\widetilde{H}_{1,1}$ оператор $\widetilde{C}_{2}$ обратим в силу обратимости операторов $\widetilde{Q}$ и $\widetilde{\gamma}_{n}$. Так как эти операторы взаимно сопряжены, то $\widetilde{C}_{2}$ имеет столько же отрицательных собственных значений, сколько и оператор $C_{2}$. В частности, при выполнении условия статической устойчивости $C_{2} \gg 0$ выполнено свойство $\widetilde{C}_{2} \gg 0$. В общем случае оператор $\widetilde{C}_{2}$ имеет не более $\varkappa=2$ отрицательных собственных значений, а значит, ограничен снизу, т.е. $\widetilde{C}_{2} \gg-h I, h \geqslant 0$.

Лемма 12. Пучок (48) при $\alpha \geqslant 0$ имеет ненулевой дискретный спектр, состоящий из изолированных конечнократных собственных значений, расположенных симметрично относительно вещественной оси, с единственной предельной точкой на бесконечности как в случае статической устойчивости, так и при ее отсутствии.

Доказательство. Дискретность спектра данной оператор-функции следует из результатов А. Г. Костюченко и А. А. Шкаликова (см. $[18,19])$. В частности, в статье [19] указывается, что спектр пучка $L(\lambda)=\lambda^{2} F+\lambda G+H-V$ дискретен, если он является эллиптическим, т.е. если выполнены следующие условия: $H \gg 0$ имеет дискретный спектр; $V$ симметричен и $H$-компактен; $F \gg 0$ ограничен; $G$ симметричен и $\mathcal{D}\left(H^{1 / 2}\right) \subset \mathcal{D}(G), L(\lambda)>0$ для достаточно больших $|\lambda|$, $\lambda \in \mathbb{R}$. В задаче (48) считаем, что $\widetilde{C}_{2}=H-V$, где $H=\widetilde{C}_{2}+h I \gg 0$ имеет дискретный спектр, так как он соответствует собственным значениям задачи (37) при $\widetilde{C}_{1}=I ; V=h I$ ограничен, а потому $H$ - компактен; $F=\widetilde{C}_{1} \gg 0$ ограничен; $G=\alpha P$ ограничен, а потому $\mathcal{D}\left(H^{1 / 2}\right) \subset \mathcal{D}(\alpha P)=\widetilde{H}_{1}$. Наконец, в силу положительной определенности оператора $\widetilde{C}_{1}$ для достаточно больших по модулю вещественных $\lambda$ свойство $L_{\alpha}(\lambda)>0$ выполнено.

Лемма 13. При выполнении условия $C_{2} \geqslant 0$ спектр задачи (46)-(47) (пучка (48)) лежит в полосе

$$
0 \leqslant \operatorname{Re} \lambda \leqslant \frac{\alpha}{c_{1}}, \quad 0<c_{1} \leqslant \frac{\left(\widetilde{C}_{1} \widetilde{z}_{1}, \widetilde{z}_{1}\right)_{\widetilde{H}_{1}}}{\left\|\widetilde{z}_{1}\right\|_{\widetilde{H}_{1}}^{2}} \leqslant c_{2} .
$$

Если оператор $C_{2}$ имеет $\varkappa \leqslant 2$ отрицательных собственных значений, то задача имеет не более и собственных значений в левой комплексной полуплоскости. 
Доказательство. Уравнения (46), (47) можно записать в виде

$$
-\lambda\left(\begin{array}{cc}
\widetilde{C}_{1} & 0 \\
0 & g C_{2}
\end{array}\right)\left(\begin{array}{l}
\widetilde{z}_{1} \\
z_{2}
\end{array}\right)+\left(\begin{array}{cc}
\alpha P & 0 \\
0 & 0
\end{array}\right)\left(\begin{array}{l}
\widetilde{z}_{1} \\
z_{2}
\end{array}\right)=-g\left(\begin{array}{cc}
0 & \widetilde{Q} C_{2} \\
-C_{2} \widetilde{\gamma}_{n} & 0
\end{array}\right)\left(\begin{array}{l}
\widetilde{z}_{1} \\
z_{2}
\end{array}\right) .
$$

Отсюда в силу кососамосопряженности операторной матрицы, стоящей в правой части (49), для собственных значений получаем соотношение

$$
-\operatorname{Re} \lambda\left[\left(\widetilde{C}_{1} \widetilde{z}_{1}, \widetilde{z}_{1}\right)+g\left(C_{2} z_{2}, z_{2}\right)\right]+\alpha\left(P \widetilde{z}, \widetilde{z}_{1}\right)=0 .
$$

Если $C_{2} \geqslant 0$, то отсюда в силу положительной определенности оператора $\widetilde{C}_{1}$ получаем неравенство

$$
0 \leqslant \operatorname{Re} \lambda \leqslant \frac{\alpha\left(P \widetilde{z}, \widetilde{z}_{1}\right)}{\left(\widetilde{C}_{1} \widetilde{z}_{1}, \widetilde{z}_{1}\right)} \leqslant \frac{\alpha}{c_{1}}
$$

Собственные значения в левой комплексной полуплоскости возможны лишь на элементах, для которых $\left(C_{2} z_{2}, z_{2}\right)<0$. Такие элементы согласно лемме 2 образуют не более чем $\varkappa$-мерное подпространство, следовательно таких собственных значений может быть с учетом кратности не более чем $\varkappa$ штук.

Так как оператор $\widetilde{C}_{1} \geqslant c_{1} I, \widetilde{C}_{2} \gg-h I$, то существует константа $s \geqslant 0$ такая, что $s^{2} \widetilde{C}_{1}+$ $\widetilde{C}_{2} \gg s^{2} c_{1}-h \geqslant 0$, т.е. оператор, стоящий в левой части, является положительно определенным. В частности, если $\widetilde{C}_{2} \gg 0$, будем считать, что $s=0$.

Осуществим в пучке (48) замену спектрального параметра $\nu:=\lambda+s$, тогда

$$
\left[\left(s^{2} \widetilde{C}_{1}+\widetilde{C}_{2}+\alpha s P\right)-\nu\left(\alpha P+2 s \widetilde{C}_{1}\right)+\nu^{2} \widetilde{C}_{1}\right] \widetilde{z}_{1}=0 .
$$

Осуществляя теперь замену $\eta=\widetilde{C}_{1}^{1 / 2} \widetilde{z}_{1} \in \widetilde{H}_{1}$ и действуя на обе части оператором $\widetilde{C}_{1}^{-1 / 2}$, приходим к задаче

$$
M_{\alpha}(\nu) \eta:=\left[\widetilde{B}_{0, \alpha}-\nu \widetilde{B}_{1, \alpha}+\nu^{2} I\right] \eta=0,
$$

где

$$
\widetilde{B}_{0, \alpha}:=\widetilde{C}_{1}^{-1 / 2}\left(s^{2} \widetilde{C}_{1}+\widetilde{C}_{2}+\alpha s P\right) \widetilde{C}_{1}^{-1 / 2}, \quad \widetilde{B}_{1, \alpha}=\widetilde{C}_{1}^{-1 / 2}(\alpha P) \widetilde{C}_{1}^{-1 / 2}+2 s I .
$$

Так как $s^{2} \widetilde{C}_{1}+\widetilde{C}_{2} \gg 0$, то оператор $\widetilde{B}_{0, \alpha}$ является неограниченным положительно определенным в $\widetilde{H}_{1}$, при этом оператор $\widetilde{B}_{1, \alpha}$ ограничен и неотрицателен.

В [23, с. 111] доказано, что пучок такого типа имеет дискретный спектр, при этом система соответствующих корневых элементов двукратно полна. Доказательство основано на сведении данного пучка к пучку Келдыша с ограниченными операторными коэффициентами, см. [13].

Осуществим в задаче (50) замены переменных и линеаризацию по параметру:

$$
\nu=: i \widetilde{\nu}, \quad \widetilde{\nu} \eta=: \psi, \quad \widetilde{B}_{0, \alpha}^{1 / 2} \eta=: \varphi .
$$

Получаем равносильную задачу на собственные значения

$$
\left(\begin{array}{cc}
0 & \widetilde{B}_{0, \alpha}^{1 / 2} \\
\widetilde{B}_{0, \alpha}^{1 / 2} & -i \widetilde{B}_{1, \alpha}
\end{array}\right)\left(\begin{array}{l}
\varphi \\
\psi
\end{array}\right)=\widetilde{\nu}\left(\begin{array}{l}
\varphi \\
\psi
\end{array}\right) .
$$

Коротко ее можно переписать в виде

$$
(A+B) y=\widetilde{\nu} y, \quad y:=(\varphi ; \psi)^{\tau} \in \widetilde{H}_{1}^{2}=\widetilde{H}_{1} \oplus \widetilde{H}_{1},
$$

где

$$
A:=\left(\begin{array}{cc}
0 & \widetilde{B}_{0, \alpha}^{1 / 2} \\
\widetilde{B}_{0, \alpha}^{1 / 2} & 0
\end{array}\right), \quad B:=\left(\begin{array}{cc}
0 & 0 \\
0 & -i \widetilde{B}_{1, \alpha}
\end{array}\right) .
$$

Определение 3. Говорят, что оператор $B$ сильно $q$-подчинен оператору $A$, если он $A^{q}$-подчинен при $q \in[0 ; 1)$, т.е. если

$$
\left\|B A^{-q} x\right\| \leqslant r\|x\|, \quad \forall x \in H
$$


Определение 4. Будем говорить, следуя В. Б. Лидскому (см., например, [1], а также [21]), что система корневых элементов $f_{k}$ несамосопряженного оператора $A$ образует в гильбертовом пространстве $H$ базис Абеля-Лидского со скобками порядка $\alpha$, если весь спектр оператора $A$, кроме возможно конечного числа собственных значений, лежит в объединении конечного числа открытых лучей с растворами не превосходящими $\theta$, где $\alpha \theta<\pi$. При этом формальный ряд из корневых элементов $f(t):=\sum_{k} c_{k}(t) f_{k}$, где

$$
c_{k}(t)=\frac{1}{2 \pi i} \oint_{\gamma_{k}} e^{-t \lambda^{\alpha}}(\lambda I-A)^{-1} f d \lambda
$$

(контур $\gamma_{k}$ окружает одно изолированное собственное значение оператора $A$ ) сходится к данному элементу $f \in H$ после некоторой расстановки скобок (не зависящей от выбора элемента $f$ ) при $t \rightarrow+0$. Отметим, что ветвь функции $\lambda^{\alpha}$ в каждом из углов, содержащих спектр, выбирается так, чтобы функция $e^{-\lambda^{\alpha} t}$ при $t>0$ экспоненциально убывала в этом угле, когда $|\lambda| \rightarrow \infty$.

Теорема 4. Система корневых элементов задачи (53) образует базис Абеля-Лидского со скобками порядка $\alpha>3$ в пространстве $\widetilde{H}_{1}^{2}$.

Доказательство. Несложно заметить, что неограниченный самосопряженный оператор $A$ имеет те же собственные значения, что задача $\widetilde{B}_{0, \alpha} \eta=-\nu^{2} \eta=\widetilde{\nu}^{2} \eta$. При этом в силу определения оператора $\widetilde{B}_{0, \alpha}$ и того факта, что $\widetilde{C}_{1}$ ограничен и ограниченно обратим, получаем, что $\widetilde{B}_{0, \alpha}$ так же, как и $\widetilde{C}_{2}$ имеет дискретный спектр с асимптотикой $\widetilde{\nu}_{k}^{2} \sim \mu_{k} \sim c k^{1 / 2}$, отсюда собственные значения оператора $A$ имеют асимптотику $\widetilde{\nu}_{k}^{ \pm} \sim \pm \sqrt{\mu_{k}} \sim \pm \sqrt{c} k^{1 / 4}$, т.е. выполнена оценка $\left|\widetilde{\nu}_{k}\right| \leqslant \widetilde{c} k^{p}$ для $p=1 / 4$. При этом оператор $B$ вслед за $\widetilde{B}_{1, \alpha}$ ограничен и потому является сильно $q$-подчиненным оператору $A$ при $q=0$.

Отсюда утверждение теоремы для $\alpha>p^{-1}-(1-q)=3$ следует из теорем А. С. Маркуса и В. Э. Кацнельсона (см. [12,22], а также [1]) в силу выполнения условия $p(1-q)=1 / 4<1$.

Теорема 5. Собственные значения задачи (48) находятся в замыкании обдединения равных кругов радиуса $r=\left\|\widetilde{B}_{1, \alpha}\right\| \leqslant \alpha c_{1}^{-1}+2 s$ с иентрами в точках $z_{k}= \pm i \mu_{k}^{1 / 2}$, отвечающих собственным значениям невозмущенной задачи при $\alpha=0$. В частности, если $\widetilde{C}_{2} \gg 0$, то все собственные значения локализованы вдоль мнимой оси и равномерно сходятся $к$ собственным значениям невозмущенной задачи при $\alpha \rightarrow 0$.

Доказательство. Действительно, пусть $\nu \in \rho(A)$, тогда

$$
(A+B)-\nu I=\left(I+B R_{A}(\nu)\right)(A-\nu I) .
$$

Так как оператор $A$ самосопряжен и имеет дискретный спектр, то

$$
\left\|R_{A}(\nu)\right\|=\sup _{\nu_{j} \in \sigma(A)}\left|\nu_{j}-\nu\right|^{-1} .
$$

Следовательно, оператор $I+B R_{A}(\nu)$ обратим (обратный строится в виде ряда Неймана), если при всех $j$ выполнена оценка

$$
\|B\| \cdot \sup _{\nu_{j} \in \sigma(A)}\left|\nu_{j}-\nu\right|^{-1}<1,
$$

т.е. любое число $\nu \in \mathbb{C}$, удовлетворяющее неравенствам

$$
\left|\nu-\nu_{j}\right|>r\left(\nu_{j} \in \sigma(A)\right),
$$

лежит в $\rho(A+B)$, если

$$
r:=\|B\|=\left\|\widetilde{B}_{1, \alpha}\right\| \leqslant \alpha\left\|\widetilde{C}_{1}^{-1 / 2} P \widetilde{C}_{1}^{-1 / 2}\right\|+2 s \leqslant \alpha c_{1}^{-1}+2 s .
$$

Таким образом доказано, что спектр оператора $A+B$ лежит в замыкании объединения равных кругов радиуса $r$ с центрами в собственных значениях $\nu_{k}$ оператора $A$. Возвращаясь по заменам к собственным значениям $\lambda$, получаем утверждение теоремы. 
Применяя к пучку $M_{\alpha}(\nu)$ лемму 13 , несложно заключить, что собственные значения пучка локализованы в полосе

$$
0 \leqslant \operatorname{Re} \nu \leqslant\left\|\widetilde{B}_{1, \alpha}\right\| \leqslant \alpha c_{1}^{-1}+2 s
$$

отсюда получаем оценку для собственных значений пучка (48) в случае, когда оператор $\widetilde{C}_{2}$ не знакоопределен:

$$
-s \leqslant \operatorname{Re} \lambda \leqslant \alpha c_{1}^{-1}+s .
$$

Отметим, что вопрос о числе собственных значений спектральной задачи, расположенных в левой комплексной полуплоскости, в случае статической неустойчивости при учете трения в шарнире остается открытым.

Приведенные в этом пункте результаты переносятся (при соответствующем выборе пространств и операторов) на общую задачу о колебаниях системы сочлененных тел с полостями, содержащими идеальные жидкости, в случае, когда учитывается трение в шарнирах.

\section{СПИСОК ЛИТЕРАТУРЫ}

1. Агранович M. С. О суммируемости рядов по корневым векторам несамосопряженных эллиптических операторов// Функц. анал. прилож. - 1976. - 10, № 3. - С. 1-12.

2. Азизов Т. Я., Иохвидов И. С. Основы теории линейных операторов в пространствах с индефинитной метрикой. - М.: Наука, 1986.

3. Азизов Т. Я., Копачевский Н. Д. Приложения индефинитной метрики. - Симферополь, 2014.

4. Батыр Э. И. Малые движения системы последовательно сочлененных тел с полостями, содержащими идеальную несжимаемую жидкость// Уч. зап. Таврич. нац. ун-та им. В. И. Вернадского. - 2002. 15 (54), № 2. - C. 5-10.

5. Батыр Э. И., Копачевский Н. Д. Малые движения и нормальные колебания системы сочлененных гиростатов// Совр. мат. Фундам. напр. - 2013. - 49. - С. 5-88.

6. Бирман М. Ш., Соломяк М. З. Асимптотика спектра дифференциальных уравнений// Итоги науки и техн. Мат. анал. - 1977. - 14. - С. 5-52.

7. Войтицкий В. И., Копачевский Н. Д. О малых колебаниях системы из трех сочлененных маятников с полостями, заполненными несмешивающимися несжимаемыми жидкостями// Мат. Междунар. конф. «Современные методы и проблемы математической гидродинамики» (Воронеж, 3-8 мая 2018 г.), 2018. - C. 84-91.

8. Войтицкий В. И., Копачевский Н. Д. О малых движениях физического маятника, содержащего полость, заполненную системой однородных несмешивающихся жидкостей// Сб. мат. междунар. конф. «XXIX Крымская осенняя математическая школа-симпозиум по спектральным и эволюционным задачам (КРОМШ-2018)». - Симферополь, 2018. - С. 58-62.

9. Вулис И. Л., Соломяк М. З. Спектральная асимптотика вырождающейся задачи Стеклова// Вестн. ЛГУ. - 1973. - 19. - С. 148-150.

10. Вулис И. Л., Соломяк М. З. Спектральная асимптотика вырождающихся эллиптических операторов второго порядка// Изв. АН СССР. Сер. мат. - 1974. - 38, № 6. - С. 1362-1392.

11. Жуковский H. Е. О движении твердого тела, имеющего полости, наполненные однородной капельной жидкостью// в кн.: Избранные сочинения. - М.-Л.: Гостехиздат, 1948. - С. 31-52.

12. Кацнельсон В. Э. Об условиях базисности системы корневых векторов некоторых классов операторов// Функц. анал. прилож. - 1967. - 2. - С. 39-51.

13. Келдыш М. В. О полноте собственных функций нектороых классов несамосопряженных линейных операторов// Усп. мат. наук. - 1971. - 24, № 4 (160). - С. 15-41.

14. Копачевский Н. Д. О колебаниях тела с полостью, частично заполненной тяжелой идеальной жидкостью: теоремы существования, единственности и устойчивости сильных решений// в кн.: Проблеми динаміки та стійкості багатовимірних систем. - Ін-т мат. НАН України., 2005. - С. 158-194.

15. Копачевский Н. Д., Войтицкий В. И. О колебаниях сочлененных маятников с полостями, заполненными однородными жидкостями // Совр. мат. Фундам. напр. - 2019. - 65, № 3. - С. 434-512.

16. Копачевский Н. Д., Войтицкий В. И., Ситшаева З. З. О колебаниях двух сочлененных маятников, содержащих полости, частично заполненные несжимаемой жидкостью// Совр. мат. Фундам. напр. 2017. - 63, № 4. - С. 627-677.

17. Копачевский Н. Д., Крейн С. Г., Нго Зуй Кан Операторные методы в линейной гидродинамике: Эволюционные и спектральные задачи. - М.: Наука, 1989. 
18. Костюченко А. Г., Шкаликов А. А. Самосопряженные квадратичные пучки операторов и эллиптические задачи // Функц. анал. прилож. - 1983. - 17, № 2. - С. 38-61.

19. Костюченко А. Г., Шкаликов А. А. Задачи дифракции для периодической поверхности для уравнения Гельмгольца// в кн.: Избранные вопросы математики и механики и их приложения. - М.: Изд-во МГУ, 1999. - С. 240-258.

20. Крейн С. Г., Моисеев Н. Н. О колебаниях твердого тела, содержащего жидкость со свободной границей// Прикл. мат. мех. - 1957. - 21, № 2. - С. 169-174.

21. Лидский В. Б. О суммируемости рядов по главным векторам несамосопряженных операторов// Тр. Моск. мат. о-ва. - 1962. - С. 3-35.

22. Маркус А. С. О разложении по корневым векторам слабо возмущенного самосопряженного оператора// Докл. АН СССР. - 1962. - 142, № 3. - С. 538-541.

23. Маркус A. C. Введение в спектральную теорию полиномиальных операторных пучков. - Кишинев: Штиинца, 1986.

24. Микишев Г. Н., Рабинович Б. И. Динамика твердого тела с полостями, частично заполненными жидкостью. - М.: Машиностроение, 1968.

25. Моисеев Н. Н. Движение твердого тела, имеющего полость, частично заполненную идеальной капельной жидкостью// Докл. АН СССР. - 1952. - 85, № 4. - С. 719-722.

26. Моисеев Н. Н. Задача о движении твердого тела, содержащего жидкие массы, имеющие свободную поверхность// Мат. сб. - 1953. - 32, № 1. - С. 61-96.

27. Моисеев H. Н., Петров А. А. Численные методы расчета собственных частот и колебаний ограниченного объема жидкости. - М.: Изд-во ВЦ АН СССР, 1966.

28. Моисеев Н. Н., Румяниев В. В. Динамика тела с полостями, содержащими жидкость. - М.: Наука, 1965.

29. Нариманов Г. С. О движении твердого тела, полость которого частично заполнена жидкостью// Прикл. мат. мех. - 1956. - 20, № 1. - С. 21-38.

30. Охоцимский Д. Е. К теории движения тела с полостями, частично заполненными жидкостью// Прикл. мат. мех. - 1956. - 20, № 1. - С. 3-20.

31. Понтрягин Л. С. Эрмитовы операторы в пространстве с индефинитной метрикой// Изв. АН СССР. Сер. мат. - 1944. - 8, № 6. - С. 243-280.

32. Panonopm И. М. Колебания упругой оболочки, частично заполненной жидкостью. - М.: Машиностроение, 1967.

33. Сретенский Л. Н. Колебание жидкости в подвижном сосуде// Изв. АН СССР. Техн. науки. - 1951. - 10. - C. 1483-1494.

34. Фещенко С. Ф., Луковский И. А., Рабинович Б. И., Докучаев Л. В. Методы определения присоединенных масс жидкости в подвижных областях. - Киев: Наукова думка, 1969.

35. Черноусъко Ф. Л. Движение твердого тела с полостями, содержащими вязкую жидкость. - М.: Изд-во ВЦ АН CCCP, 1968.

36. Kopachevsky N. D., Krein S. G. Operator Approach to Linear Problems of Hydrodynamics. Self-adjoint Problems for an Ideal Fluid. - Basel-Boston-Berlin: Birkhäuser, 2001.

37. Kopachevsky N. D., Krein S. G. Operator Approach to Linear Problems of Hydrodynamics. Nonself-adjoint Problems for Viscous Fluids. - Basel-Boston-Berlin: Birkhäuser, 2003.

\section{Войтицкий Виктор Иванович}

Таврическая академия

Крымского федерального университета им. В. И. Вернадского, Симферополь

E-mail: victor.voytitsky@gmail.com

Копачевский Николай Дмитриевич

Таврическая академия

Крымского федерального университета им. В. И. Вернадского, Симферополь

E-mail: kopachevsky@list.ru 